\title{
Control of Multiagent Systems: A Stochastic Pinning Viewpoint
}

\author{
Guoliang Wang \\ School of Information and Control Engineering, Liaoning Shihua University, No. 1 West Dandong Road, Wanghua District, \\ Fushun, Liaoning 113001, China \\ Correspondence should be addressed to Guoliang Wang; gliangwang@aliyun.com
}

Received 3 December 2013; Accepted 24 February 2014; Published 7 April 2014

Academic Editor: Hao Shen

Copyright (C) 2014 Guoliang Wang. This is an open access article distributed under the Creative Commons Attribution License, which permits unrestricted use, distribution, and reproduction in any medium, provided the original work is properly cited.

A stochastic pinning approach for multiagent systems is developed, which guarantees such systems being almost surely stable. It is seen that the pinning is closely related to being a Bernoulli variable. It has been proved for the first time that a series of systems can be stabilized by a Brownian noise perturbation in terms of a pinning scheme. A new terminology named "stochastic pinning control" is introduced to describe the given pinning algorithm. Additionally, two general cases that the expectation of the Bernoulli variable with bounded uncertainty or being unknown are studied. Finally, two simulation examples are provided to demonstrate the effectiveness of the proposed methods.

\section{Introduction}

Due to the broad applications in cooperative control of unmanned air vehicles, formation control of mobile robots, sensor networks, and cooperative surveillance, multiagent problems have drawn a lot of attention. In particular, multiagent coordination with multiple leaders becomes more and more important, which forces a group of agents into a specific target region. Because of the spatial distribution of actuators and sensors, it is of high cost or even impossible in practice to implement a centralized controller. Instead, distributed control emerged to be a promising tool for coordination of multiagent systems, which is usually to design a controller to every subsystem. During the past years, many important results have been reported in [1-6]. Many natural and manmade systems, such as ecosystems, internet, Word Wide Web, social networks, and power grids, are described by it. In recent years, the analysis and control of complex behaviors in complex networks have become a hot topic across many fields such as in [7-11]. Especially, synchronization related to being the most important collective behavior of complex networks, such as ER random, small-world, and scale-free complex networks [12-14], has been extensively studied. Via introducing a Bernoulli stochastic variable describing the random switching of controllers, the distributed synchronization of complex networks was studied in $[15,16]$.
Due to the complexity of complex networks, it is usually not easy to control a complex network by adding controllers to all nodes. Instead, pinning control only uses a small number of controllers. In this sense, it is said that pinning control is a promising method, which can efficiently reduce the number of controllers. The pinning control strategy for linear coupled networks was investigated in [17, 18], in which two different pinning strategies, random pinning and special pinning methods, were theoretically and numerically compared. During the past decades, a lot of results on synchronization of various complex networks by pinning control have emerged, for example, [19-25]. By searching such references on pinning control, it is concluded that all the pinning methods are realized by a kind of regular controllers in terms of in the drift section of a system. However, it is possible to design a controller to stabilize a stochastic system almost surely which is unable to be stabilized in mean-square sense. Based on these facts, it is asked that can we design a pinning controller referred to be a Brownian noise perturbation to stabilize multiagent systems? To the best of authors' knowledge, the control problem of multiagent systems via a pinning controller only in the diffusion part has not yet been investigated, which motivates the current research.

In this paper, the control problem of multiagent systems is firstly considered by a stochastic pinning viewpoint. In 
contrast to the existing results of pinning control methods, the main contributions of this paper are as follows. (1) The control of multiagent systems is firstly realized by a pinning control method in terms of a Brownian noise perturbation. (2) In order to achieve this goal, new pinning control in terms of stochastic pinning control (SPC) is developed, in which the Bernoulli variable plays an important role in SPC. (3) More general cases such as the expectation of Bernoulli variable with uncertainty and being unknown are considered respectively. (4) The relationship among the expectation, the pinning fraction, and the pinning control gain for both random and special pinning control is demonstrated in detail.

Notation. $\mathbb{R}^{n}$ denotes the $n$ dimensional Euclidean space; $\mathbb{R}^{m \times n}$ is the set of all $m \times n$ real matrices. $|\cdot|$ denotes the Euclidean norm. $\mathscr{E}\{\cdot\}$ is the expectation operator with respect to some probability measure. $\otimes$ is the Kronecker product. In symmetric block matrices, we use " $*$ " as an ellipsis for the terms induced by symmetry, $\operatorname{diag}\{\cdots\}$ for a block-diagonal matrix. $\mathbb{S} \triangleq\{1,2, \ldots, N\}=\mathbb{S}_{l} \bigcup \overline{\mathbb{S}}_{l}$, where $\mathbb{S}_{l}=\{1,2, \ldots, l\}$ and $\overline{\mathbb{S}}_{l}=\mathbb{S}-\mathbb{S}_{l}$.

\section{Problem Formulation}

Consider a multiagent system consisting of $N$ agents, such as in [6], the model of agent, $i \in \mathbb{S}$, is described as

$$
\dot{x}_{i}=f_{i}\left(x_{i}, t\right) \text {, }
$$

where $x_{i} \in \mathbb{R}^{n}$ is the state vector, $f_{i}\left(x_{i}, t\right): \mathbb{R}^{n} \times \mathbb{R} \rightarrow \mathbb{R}^{n}$ is the inherent nonlinear dynamic. However, it can be also be dealt with by the fuzzy method [26, 27]. In this paper, the nonlinear term $f_{i}\left(x_{i}, t\right)$ is treated directly, and an assumption is needed here.

Assumption 1. Nonlinear function $f_{i}\left(x_{i}, t\right)$ is assumed to satisfy the following condition:

$$
x_{i}^{T} f_{i}\left(x_{i}, t\right) \leq \theta_{i} x_{i}^{T} x_{i}, \quad \forall x_{i} \in \mathbb{R}^{n}, \forall t \geq 0,
$$

where $\theta_{i} \geq 0$ is a given constant.

In this paper, a new pinning control method for system (1) in terms of stochastic pinning control is proposed as

$$
\begin{gathered}
u_{i}=c \sum_{j=1}^{N} g_{i j} x_{j}-c k_{i} x_{i}, \quad i \in \mathbb{S}_{l}, \\
u_{i}=c \sum_{j=1}^{N} g_{i j} x_{j}-c \alpha(t) k_{i} x_{i}, \quad i \in \overline{\mathbb{S}}_{l},
\end{gathered}
$$

respectively, where $c$ is the coupling strength, $G=\left(g_{i j}\right) \epsilon$ $\mathbb{R}^{N \times N}$ is the coupling matrix with $g_{i j} \geq 0, i \neq j, g_{i i}=$ $-\sum_{j=1, j \neq i}^{N} g_{i j}$, which is an irreducible matrix, and $k_{i} \in \mathbb{R}^{n \times n}$ is the control gain. Bernoulli variable $\alpha(t)$ in (4) is described as

$$
\alpha(t)=1 \text { or } 0
$$

whose expectation is $\mathscr{E}\{\alpha(t)\}=\alpha$.

In this paper, such controllers will be used as a pinning controller in terms of Brownian noise perturbation. As a result, we have the closed-loop system as

$$
\begin{gathered}
d x_{i}=f_{i}\left(x_{i}, t\right) d t+c\left(\sum_{j=1}^{N} g_{i j} x_{j}-k_{i} x_{i}\right) d \omega(t), \quad i \in \mathbb{S}_{l}, \\
d x_{i}=f_{i}\left(x_{i}, t\right) d t+c\left(\sum_{j=1}^{N} g_{i j} x_{j}-\alpha(t) k_{i} x_{i}\right) d \omega(t), \\
i \in \overline{\mathbb{S}}_{l},
\end{gathered}
$$

where $\omega(t) \in \mathbb{R}^{n}$ is a $n$-dimensional Brownian motion or Wiener process. Let $x=\left[\begin{array}{lll}x_{1}^{T} & \cdots & x_{N}^{T}\end{array}\right]^{T}, f=\left[\begin{array}{lll}f_{1}^{T} & \cdots & f_{N}^{T}\end{array}\right]^{T}$, and $K_{\alpha(t)}=\operatorname{diag}\left\{k_{1}, \ldots, k_{l}, \alpha(t) k_{l+1}, \ldots, \alpha(t) k_{N}\right\}$; one has $G_{1}=G-K_{\alpha(t)=1}$ and $G_{2}=G-K_{\alpha(t)=0}$, respectively. Without of loss generality, in this paper it is assumed that $k_{i}=k_{j}=k>0$, for all $i, j \in \mathbb{S}$. Based on these notations, we have

$$
d x=f(x, t) d t+c \widehat{G}(\eta(t)) x d \omega(t),
$$

where $\widehat{G}(\eta(t))=G(\eta(t)) \otimes I_{n}$. Operation mode $\{\eta(t), t \geq 0\}$ takes values in set $\mathbb{B}=\{1,2\}$ and is described as

$$
\eta(t)= \begin{cases}1, & \text { if } \alpha(t)=1 \\ 2, & \text { if } \alpha(t)=0,\end{cases}
$$

whose probabilities are $\operatorname{Pr}\{\eta(t)=1\}=\alpha$ and $\operatorname{Pr}\{\eta(t)=2\}=$ $1-\alpha$, respectively.

Remark 2. It is worth pointing out that the proposed pinning control method is different from the existing pinning methods. Firstly, the pinning problem of this paper is realized by a Brownian noise perturbation, which cannot be solved by the usual analysis methods. Secondly, the introduced Bernoulli variable $\alpha(t)$ plays an important role in achieving the pinning control in terms of Brownian noise control. Such differences embody the property of stochastic pinning control. That is, when $\alpha(t)=0$, only controller (6) works and is a pinning controller. On the contrary, if $\alpha(t)=1$, the desired controller becomes a distributed controller, which is not pinning control in fact. In this sense, it is said that the developed pinning control is a stochastic control method.

Remark 3. It should be remarked that this framework is necessary to achieve pinning control through Brownian noise perturbations. If there is no $\alpha(t)$ in (7), the underlying systems become (6) with $i \in \mathbb{S}_{l}$ and (1) with $i \in \overline{\mathbb{S}}_{l}$, which can be obtained by applying the usual pinning methods directly. Unfortunately, it is concluded that this pinning framework is very hard to realize the desired object. 
The reason will be explained later. On the other hand, when $\alpha(t) \equiv 1$, we have (6) only, which is seen as a distributed controller instead of a pinning controller. Thus, it is claimed that the given pinning control algorithm bridges the traditional pinning control and distributed control. In addition, $K_{\alpha(t)}$ can also choose other forms, such as $K_{\alpha(t), \beta(t)}=$ $\operatorname{diag}\left\{\alpha(t) k_{1}, \ldots, \alpha(t) k_{l}, \beta(t) k_{l+1}, \ldots, \beta(t) k_{N}\right\}$, where both $\alpha(t)$ and $\beta(t)$ are dependent or independent Bernoulli variables.

Definition 4. The equilibrium of system (8) is said to be almost surely exponentially stable if for any $x_{0} \in \mathbb{R}^{n}$

$$
\lim _{t \rightarrow \infty} \sup \frac{1}{t} \log \left(\left|x\left(t, x_{0}\right)\right|\right)<0 \quad \text { a.s. }
$$

\section{Main Results}

Theorem 5. For given scalars $\theta$ and $\alpha$, the equilibrium of system (8) is almost surely exponentially stable, if there exists $k>0$ such that

$$
2 \theta+\alpha c^{2} \Omega_{1}+(1-\alpha) c^{2} \Omega_{2}<0
$$

where $\Omega_{i}=\lambda_{\max }\left(G_{i}^{T} G_{i}\right)-2 \lambda_{\max }^{2}\left(G_{i}\right), i \in \mathbb{B}$.

Proof. For any given initial condition $x_{0} \neq 0$, it is known that $x(t) \triangleq x\left(t ; x_{0}\right)$ will never reach zero with probability one, and by Itô formula, it is obtained that

$$
\begin{aligned}
d & {\left[\log \left(|x|^{2}\right)\right] } \\
= & \frac{\left(2 x^{T} f(x, t)+c^{2} x^{T} \widehat{G}_{i}^{T} \widehat{G}_{i} x\right) d t+2 c x^{T} \widehat{G}_{i} x d \omega(t)}{|x|^{2}} \\
& -\frac{2 c^{2}\left|x^{T} \widehat{G}_{i} x\right|^{2}}{|x|^{4}} d t \\
= & \frac{\left(2 x^{T} f(x, t)+c^{2} x^{T}\left(G_{i}^{T} G_{i}\right) \otimes I_{n} x\right) d t+2 c x^{T} \widehat{G}_{i} x d \omega(t)}{|x|^{2}} \\
& -\frac{2 c^{2}\left|x^{T} \widehat{G}_{i} x\right|^{2}}{|x|^{4}} d t \\
\leq & \left(2 \theta+c^{2} \Omega_{i}\right) d t+\frac{2 c x^{T} \widehat{G}_{i} x}{|x|^{2}} d \omega(t) .
\end{aligned}
$$

Then, it is obtained that

$$
\begin{aligned}
& \log \left(|x(t)|^{2}\right) \leq \log \left(\left|x_{0}\right|^{2}\right) \\
& \quad+\int_{0}^{t}\left(2 \theta+c^{2} \Omega(\eta(s))\right) d s+M(t),
\end{aligned}
$$

where $M(t)=\int_{0}^{t}\left(\left(2 c x^{T}(s) \widehat{G}(\eta(s)) x(s)\right) /|x(s)|^{2}\right) d \omega(s)$ is a continuous martingale vanishing at $t=0$. Taking into account (9), it is seen that

$$
\begin{aligned}
\lim _{t \rightarrow \infty} & \frac{1}{t} \int_{0}^{t}\left(2 \theta+c^{2} \Omega(\eta(s))\right) d s \\
& =2 \theta+\alpha c^{2} \Omega_{1}+(1-\alpha) c^{2} \Omega_{2} \quad \text { a.s. }
\end{aligned}
$$

On the other hand, it is concluded that for any finite $k$ given in $G_{i}$, there exists a positive scalar $\rho_{i}$ that the quadratic variation of $M(t)$ is

$$
\begin{aligned}
\langle M(t), M(t)\rangle & =\int_{0}^{t} \frac{4 c^{2}\left|x^{T}(s) \widehat{G}(\eta(s)) x(s)\right|^{2}}{|x(s)|^{4}} d s \\
& \leq 4 t \max _{i \in \mathbb{B}} \rho_{i} .
\end{aligned}
$$

Applying the strong law of the large numbers to $M(t)$, one has

$$
\lim _{t \rightarrow \infty} \frac{M(t)}{t}=0 \quad \text { a.s. }
$$

Based on (14) and (16), we conclude that

$$
\begin{aligned}
& \lim _{t \rightarrow \infty} \sup \frac{1}{t} \log \left(\left|x\left(t, x_{0}\right)\right|\right) \\
& \quad \leq 2 \theta+\alpha c^{2} \Omega_{1}+(1-\alpha) c^{2} \Omega_{2}<0 \quad \text { a.s. }
\end{aligned}
$$

which is guaranteed by (11). This completes the proof.

Remark 6. By Theorem 5, it is known that if condition (11) holds, one can stabilize system (1) by a pinning control tactic in terms of Brownian noise perturbation. However, condition (11) is impossible or hard to be satisfied if controller (7) is with $\alpha(t) \equiv 0$. If $\alpha(t) \equiv 0$, condition (11) becomes $2 \theta+c^{2} \Omega_{2}<0$, where pinning controller is same as the traditional pinning controller. Unfortunately, it is said that $2 \theta+c^{2} \Omega_{2}<0$ with $\theta>0$ is impossible or hard to be satisfied because of $\Omega_{2}>$ 0 . That is, due to the property of $G$ in (3) or (4), we have $\lambda_{\text {max }}\left(G_{2}\right)<0$, and $\lambda_{\text {max }}^{2}\left(G_{2}\right)=\left(\lambda_{\max }\left(G_{2}\right)\right)^{2}$. It usually results in $\lambda_{\max }\left(G_{2}^{T} G_{2}\right) \gg 2 \lambda_{\text {max }}^{2}\left(G_{2}\right)$. In this sense, when $\alpha(t) \equiv 0$, it is difficult to realize the pinning control goal of this paper.

From Theorem 5, it is seen that the expectation $\alpha$ plays an important role in SPC which should be given exactly. In some applications, it is very hard or of high cost to obtain $\alpha$ exactly. Instead, only its estimation $\tilde{\alpha}$ is available. Then, it is natural and important to study how to realize SPC when $\alpha$ is uncertain. If there exists an uncertainty in $\alpha$, we will use its estimation $\widetilde{\alpha}$. It is described as

$$
\Delta \alpha=\alpha-\tilde{\alpha}, \quad \tilde{\alpha} \in[0,1]
$$

where admissible uncertainty $\Delta \alpha \in[-\epsilon, \epsilon]$ with $\epsilon \in[0,1]$. Then, we have the following theorem. 
Theorem 7. For given scalars $\theta$ and $\tilde{\alpha}$, the equilibrium of system (8) is robust almost surely exponentially stable for any admissible uncertainty (18), if there exist $k>0$ and $\mu>0$ such that

$$
\begin{aligned}
2 \theta & +\widetilde{\alpha} c^{2} \Omega_{1}+(1-\widetilde{\alpha}) c^{2} \Omega_{2}+0.25 \epsilon^{2} c^{4} \mu \\
& +\left(\Omega_{1}-\Omega_{2}\right)^{2} \mu^{-1}<0 .
\end{aligned}
$$

Proof. Based on the proof of Theorem 5, it is obtained that the change of $\alpha$ only takes place in (14); that is

$$
\begin{aligned}
& \alpha c^{2} \Omega_{1}+(1-\alpha) c^{2} \Omega_{2} \\
& \quad=\tilde{\alpha} c^{2} \Omega_{1}+(1-\tilde{\alpha}) c^{2} \Omega_{2}+\Delta \alpha c^{2}\left(\Omega_{1}-\Omega_{2}\right)<0 .
\end{aligned}
$$

For $\Delta \alpha c^{2}\left(\Omega_{1}-\Omega_{2}\right)$ with any $\mu>0$, it is seen that

$$
\Delta \alpha c^{2}\left(\Omega_{1}-\Omega_{2}\right) \leq 0.25(\Delta \alpha)^{2} c^{4} \mu+\left(\Omega_{1}-\Omega_{2}\right)^{2} \mu^{-1} .
$$

Taking into account (19) and (21), one has (11). That completes the proof.

It is seen that the conditions of Theorems 5 and 7 are not LMIs, which are not solved directly. In the following, another condition in terms of LMIs with equation constraints is proposed, which could be solved easily.

Theorem 8. For given scalars $\theta$ and $\alpha$, the equilibrium of system (8) is almost surely exponentially stable, if there exist $k>0, \delta_{i}>0, \gamma_{i}>0, \bar{\gamma}_{i}>0, \sigma_{i}>0$, and $\bar{\sigma}_{i}>0$, such that the following LMIs hold for all $i \in \mathbb{B}$ :

$$
\begin{gathered}
2 \theta+\alpha c^{2} \bar{\Omega}_{1}+(1-\alpha) c^{2} \bar{\Omega}_{2}<0 \\
{\left[\begin{array}{cc}
-\delta_{i} I & G_{i}^{T} \\
G_{i} & -I
\end{array}\right] \leq 0} \\
{\left[\begin{array}{cc}
-\bar{\sigma}_{i} & \bar{\gamma}_{i} \\
\bar{\gamma}_{i} & -1
\end{array}\right] \leq 0} \\
\gamma_{i} \bar{\gamma}_{i}=1, \quad \sigma_{i} \bar{\sigma}_{i}=1
\end{gathered}
$$

either

$$
G_{i}+G_{i}^{T} \geq 2 \gamma_{i} I
$$

or

$$
G_{i}+G_{i}^{T} \leq-2 \gamma_{i} I
$$

where $\bar{\Omega}_{i}=\delta_{i}-2 \sigma_{i}$.
Proof. Based on (11), it is known that if there are $\delta_{i}>0$ and $\gamma_{i}>0$ such that

$$
\begin{gathered}
\lambda_{\max }\left(G_{i}^{T} G_{i}\right) \leq \delta_{i}, \\
\lambda_{\max }^{2}\left(G_{i}\right) \geq \gamma_{i}^{2},
\end{gathered}
$$

hold, which are guaranteed by

$$
\begin{gathered}
G_{i}^{T} G_{i} \leq \delta_{i} I \\
\lambda_{\max }\left(G_{i}\right) \geq \gamma_{i} I
\end{gathered}
$$

or

$$
\lambda_{\max }\left(G_{i}\right) \leq-\gamma_{i} I,
$$

respectively. Based on these, it is obvious that (28) can be obtained by (23), (26), or (27). Then, we have (11) which is insured by

$$
2 \theta+\alpha c^{2}\left(\delta_{1}-2 \gamma_{1}^{2}\right)+(1-\alpha) c^{2}\left(\delta_{2}-2 \gamma_{2}^{2}\right)<0,
$$

where $\delta_{i}$ and $\gamma_{i}$ should be determined. Because of nonlinear term $\gamma_{i}^{2}$ in (31), it cannot be solved by LMI tool box directly. By introducing a variable $\sigma_{i}$ satisfying $\gamma_{i}^{2} \geq \sigma_{i}$, it is concluded that it is equivalent to $\sigma_{i}^{-1} \geq \gamma_{i}^{-2}$. By Schur complement and condition (25), one has (22)-(25) implying (11). This completes the proof.

If the expectation $\alpha$ is unknown, how to achieve a useful SPC is another general case. For this case, we have the following theorem.

Theorem 9. For a given scalar $\theta$, the equilibrium of system (8) is almost surely exponentially stable, if there exists $k>0$ such that

$$
\Omega_{1}+\Omega_{2}<0
$$

In this case, the expectation $\alpha$ can be unknown, but it should be satisfied

$$
\alpha>\frac{\Omega_{2}}{\Omega_{2}-\Omega_{1}} .
$$

Proof. Based on the proof of Theorem 5, one can easily have the equilibrium of system (8) almost surely exponentially stable, if there exists $k>0$ such that

$$
2 \theta+c^{2} \alpha \Omega_{1}+c^{2}(1-\alpha) \Omega_{2}<0,
$$

which is equivalent to

$$
2 \theta+c^{2}\left[\alpha\left(\Omega_{1}-\Omega_{2}\right)+\Omega_{2}\right]<0 .
$$

If $\alpha$ is unknown but satisfies (33), one could always choose a sufficiently large scalar $c$ such that (35) holds. This completes the proof. 
Corollary 10. For a given scalar $\theta$, the equilibrium of system (8) is almost surely exponentially stable, if there exists $k>0$ such that $\Omega_{1}<0$ and (32) hold. In this case, the expectation $\alpha$ can be unknown, but it should be satisfied $\alpha \in(0.5,1]$.

Proof. Similar to the proof of Theorem 9, (34) can be rewritten to be

$$
2 \theta+(2 \alpha-1) c^{2} \Omega_{1}+(1-\alpha) c^{2}\left(\Omega_{1}+\Omega_{2}\right)<0
$$

which could be guaranteed by

$$
\begin{gathered}
2 \theta+(2 \alpha-1) c^{2} \Omega_{1}<0 \\
(1-\alpha) c^{2}\left(\Omega_{1}+\Omega_{2}\right) \leq 0
\end{gathered}
$$

Since $\Omega_{1}<0$ and $\alpha \in(0.5,1]$, it is obtained that there is always a sufficiently large constant $c$ such that (37) holds. On the other hand, under the conditions of $\alpha$ and $c>0$, we have (33) implying (38) directly. This completes the proof.

It is claimed that the key idea of SPC described by (3) and (4) can be used to construct a pinning controller in the drift section. That is

$$
\begin{gathered}
\dot{x}_{i}=f_{i}\left(x_{i}, t\right)+c\left(\sum_{j=1}^{N} g_{i j} x_{j}-\alpha(t) k_{i} x_{i}\right), \quad i \in \mathbb{S}_{l}, \\
\dot{x}_{i}=f_{i}\left(x_{i}, t\right)+c \sum_{j=1}^{N} g_{i j} x_{j}, \quad i \in \overline{\mathbb{S}}_{l} .
\end{gathered}
$$

Let $x=\left[\begin{array}{lll}x_{1}^{T} & \cdots & x_{N}^{T}\end{array}\right]^{T}, f=\left[\begin{array}{lll}f_{1}^{T} & \cdots & f_{N}^{T}\end{array}\right]^{T}$, and $K=$ $\operatorname{diag}\left\{k_{1}, \ldots, k_{l}, 0, \ldots, 0\right\}$; one has

$$
\dot{x}=f(x, t)+c(G-\alpha(t) K) \otimes I_{n} x
$$

It is rewritten to be

$$
\dot{x}=f(x, t)+c \widetilde{G} \otimes I_{n} x+c(\alpha(t)-\alpha) K \otimes I_{n} x,
$$

where $\widetilde{G}=G+\alpha K$. Without of loss generality, in matrix $K$, it is also assumed that $k_{i}=k_{j}=k>0$, for all $i, j \in \mathbb{S}_{l}$. Since $\alpha(t)$ is a Bernoulli variable, it is known that $\mathscr{E}\{\alpha(t)-\alpha\}=0$.

Theorem 11. For given scalars $\theta$ and $\alpha$, the equilibrium of system (40) is exponentially mean-square stable, if there exists $k>0$ such that

$$
2 \theta+c \lambda_{\max }(\widetilde{G})<0
$$

Proof. Choose the following Lyapunov function:

$$
V(x(t), t)=x^{T}(t) x(t) .
$$

Based on (42), we have

$$
\begin{aligned}
\mathscr{L} V(x(t), t) & \leq x^{T}(t)\left(2 \theta I_{N n}+c \widetilde{G} \otimes I_{n}\right) x(t) \\
& \leq\left(2 \theta+c \lambda_{\max } \widetilde{G}\right) x^{T}(t) x(t)<0 .
\end{aligned}
$$

Then, there is always a sufficient small scalar $\mu>0$ such that

$$
\mathscr{L} V(x(t), t) \leq-\mu x^{T}(t) x(t)<0 .
$$

By Dynkin's formula, it is obtained that for $T>0$

$$
\begin{gathered}
\mathscr{E}\left(x^{T}(T) x(T)\right)-\mathscr{E}\left(x^{T}(0) x(0)\right) \\
\leq-\mu \int_{0}^{T} x^{T}(s) x(s) d s<0 .
\end{gathered}
$$

Applying the Gronwall-Bellman lemma to (46), one gets

$$
\mathscr{E}\left(x^{T}(t) x(t)\right) \leq|x(0)|^{2} \exp (-\mu t) .
$$

This completes the proof.

Remark 12. It should be pointed out that the pinning controller in the drift section is also different from the existing methods such as $[17,18,20,21,28]$. It is said that the pinning method of this paper is a stochastic algorithm, where the expectation plays an important role. Compared with the traditional pinning methods, the desired pinning controller is not necessary implemented online, which is added to some nodes in terms of probability $\alpha$. The correlation among the expectation $\alpha$, the pinning fraction $\delta$, and the control gain $k$ is firstly illustrated in Theorem 11 , which is also shown by numerical examples.

Similarly, when the expectation $\alpha$ is uncertain and satisfies (18), we have the following result.

Theorem 13. For given scalars $\theta$ and $\widetilde{\alpha}$, the equilibrium of system (40) is robust exponentially mean-square stable for any admissible uncertainty (18), if there exist $k>0$ and $\mu>0$ such that

$$
2 \theta+c \lambda_{\max }(\widehat{G})+c \epsilon k<0
$$

where $\widehat{G}=G+\widetilde{\alpha} K$.

Proof. Based on the proof of Theorem 11 and taking in (48), it is obtained that (44) is rewritten as

$$
\begin{aligned}
& \mathscr{L} V(x(t), t) \\
& \quad \leq x^{T}(t)\left(2 \theta I_{N n}+c \widetilde{G} \otimes I_{n}\right) x(t) \\
& \quad=x^{T}(t)\left(2 \theta I_{N n}+c \widehat{G} \otimes I_{n}+c \Delta \alpha K \otimes I_{n}\right) x(t) \\
& \quad \leq x^{T}(t)\left(2 \theta I_{N n}+c \widehat{G} \otimes I_{n}+c \epsilon k I_{N n}\right) x(t)<0,
\end{aligned}
$$

where $K$ is defined in (41). Obviously, it is known that (48) implies (49). This completes the proof.

When $\alpha$ is unknown, we have the following theorem. 


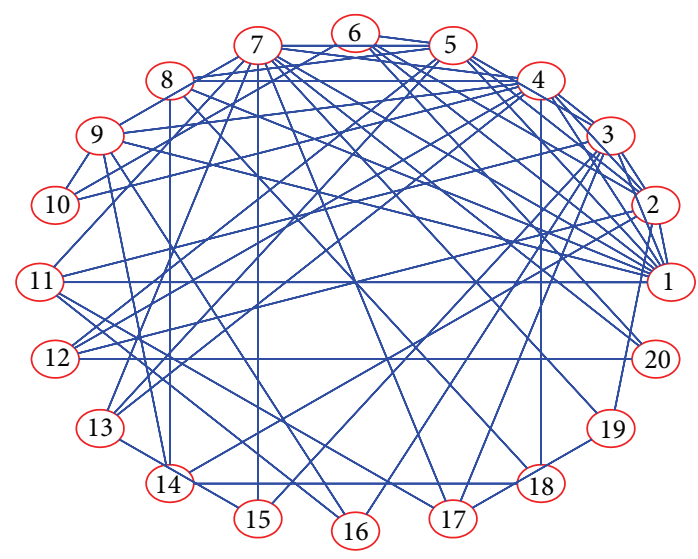

FIGURE 1: Connection of the closed-loop system.

Theorem 14. For a given scalar $\theta$, the equilibrium of system (40) is exponentially mean-square stable, if there exists $k>0$ such that

$$
\lambda_{\max }(G+K)<0 .
$$

In this case, there is an SPC such that (40) is exponentially mean-square stable with unknown $\alpha$.

Proof. Based on Theorem 11, the equilibrium of system (40) is exponentially mean-square stable, if there exists $k>0$ such that

$$
2 \theta I_{N n}+c(G+\alpha K) \otimes I_{n}<0,
$$

which is equivalent to

$$
2 \theta I_{N n}+c \alpha(G+K) \otimes I_{n}+c(1-\alpha) G \otimes I_{n}<0 .
$$

By the definition of $G$, it is known that $\lambda_{i}(G) \leq 0, i=1, \ldots, N$. Because of $c>0$ and $\alpha \in[0,1]$, it is concluded that (52) is guaranteed by

$$
2 \theta+c \alpha \lambda_{\max }(G+K)<0
$$

If (50) satisfies, one could always choose a sufficiently large scalar $c$ such that (53) holds. This completes the proof.

\section{Numerical Examples}

In this section, two numerical examples are used to demonstrate the utility of the proposed method.

Example 15. Without loss of generality, consider a multiagent system with 20 agents, whose agent is described as

$$
\begin{gathered}
\dot{x}_{1}=0.2 x_{1}+x_{2}, \\
\dot{x}_{2}=-x_{1}-0.5 x_{2} .
\end{gathered}
$$

In this example, such multiagent system will be stabilized by a stochastic pinning controller whose connection of agents in terms of scale-free network is simulated in Figure 1. Then, the coupling matrix can be obtained from Figure 1 directly.

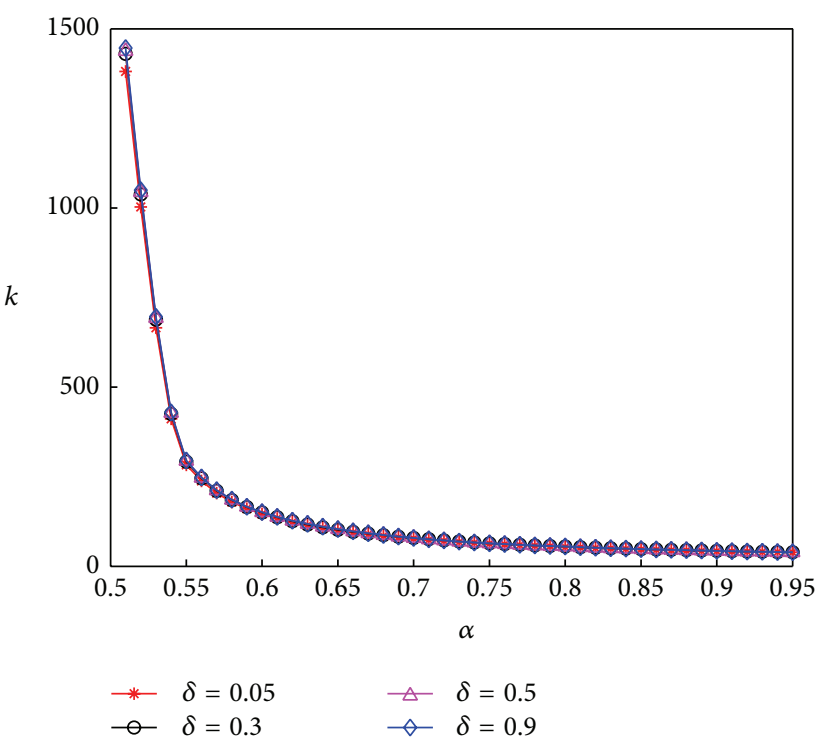

FIGURE 2: Correlation between $k$ and $\alpha$ with given different $\delta$.

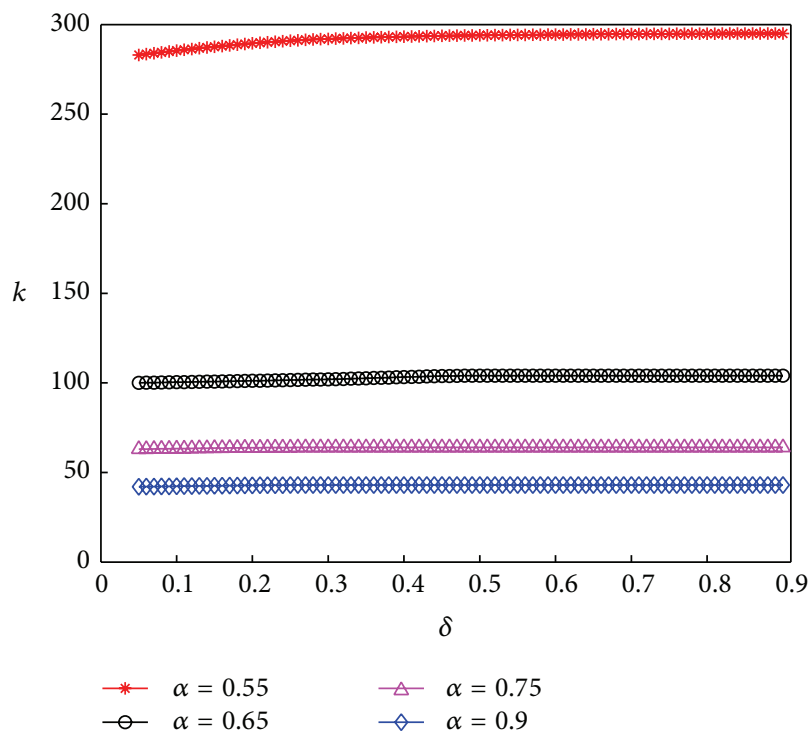

FIGURE 3: Correlation between $k$ and $\delta$ with given different $\alpha$.

From system (54), it is obtained that $\theta_{i}$ can be chosen as $\theta=1.37$. The correlations among the expectation $\alpha$, the pinning fraction $\delta$, and the pinning control gain $k$ are given in Theorem 5, which are demonstrated in Figures 2 and 3, respectively. In this paper, the special pinning control means (3) takes place in the nodes with more degrees. From Figure 2, it is seen that for given $\delta$, larger expectation $\alpha$ results in smaller control gain $k$. When $\alpha$ is small, such as $\alpha \leq 0.7$, the curve of $k$ along with $\alpha$ changes sharply, while the other section is gentle. On the other hand, the change of $k$ along with $\delta$ under given $\alpha$ is shown in Figure 3. By this simulation, one knows that larger $\delta$ results in larger $k$. Different from Figure 2, it is seen that the whole curve is gentle. If $\delta=0.1$, $\alpha=0.6$, by Theorem 5 , we have $k_{\min }=150$ with $k>0$. 


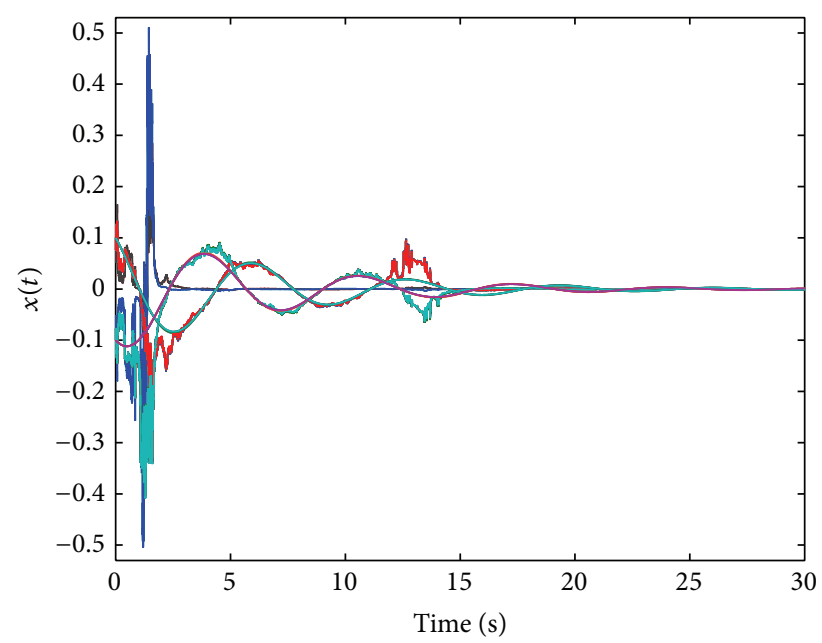

FIGURE 4: Simulation of the closed-loop system by SPC.

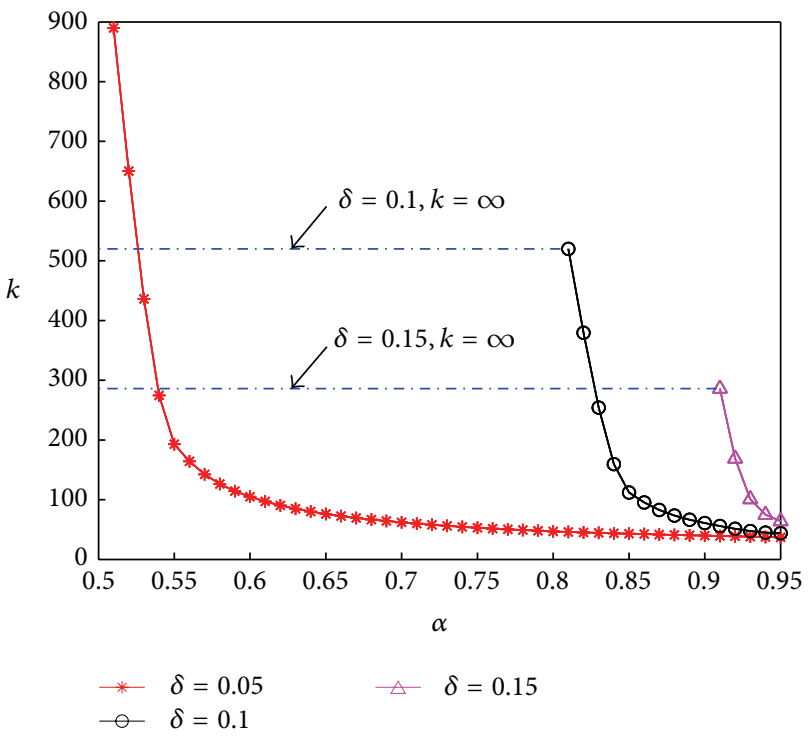

FIGURE 5: Correlation between $k$ and $\alpha$ with given different $\delta$.

Let initial condition of system (54) be $x_{0}=\left[\begin{array}{ll}0.1 & -0.1\end{array}\right]^{T}$; the state response of the closed-loop system is given in Figure 4, which is stable and demonstrates that the desired SPC is effective.

When the stochastic pinning controller is realized by random pinning control in terms of (3) taking place in any nodes, one has the following results, which are given in Figures 5 and 6, respectively. Considering Figures 2 and 5, it is concluded that control gain $k$ in both of them becomes larger when $\alpha$ takes larger values. Especially, from Figure 5, it is further obtained that in some cases with larger pinning fraction $\delta$, smaller $\alpha$ results in no solution to $k$. On the other hand, in Figure 6, one has that for some given values of $\alpha$ such as $\alpha=0.91, \alpha=0.93$ and $\alpha=0.95$, there is no solution to control gain $k$ in terms of random pinning control when $\delta$ satisfies $\delta>0.15$ and $N * \delta$ should be an integer number. On the contrary, even if $\delta=0.9$, we also have the

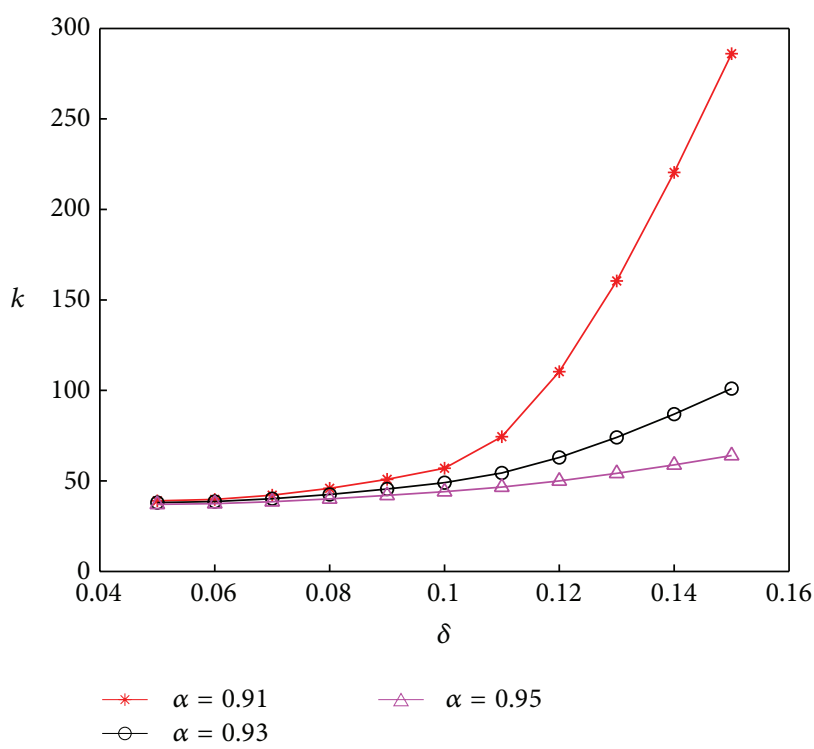

FIgURE 6: Correlation between $k$ and $\delta$ with given different $\alpha$.

control gain of special pinning controller. In this sense, it is said that special pinning control is better. For a given $\alpha$, when pinning fraction $\delta$ becomes larger, larger control gain $k$ is needed no matter which pinning control algorithm is selected. That means if one wants to pin a multiagent system by exploiting SPC described by (3) and (4) in terms of more agents controlled directly, he should provide a larger control gain $k$. Moreover, there is an interesting phenomenon in Figure 2 with $\delta=0.05$ and Figure 5 with $\delta=0.05$. That is, for the same $\alpha$, the gain of random pinning controller is smaller than one of special pinning controller. This phenomenon can be explained if the two pinning methods are effective, because of special pinning control pinning more nodes due to the controlled nodes more "important", it needs its control gain $k$ larger.

Example 16. Consider a dynamical node of complex network is a Chua's chaotic circuit described by

$$
\begin{aligned}
& \dot{x}_{1}(t)=\beta\left(-x_{1}+x_{2}-\varsigma\left(x_{1}\right)\right) \\
& \dot{x}_{2}(t)=x_{1}-x_{2}+x_{3} \\
& \dot{x}_{3}(t)=-\gamma x_{2},
\end{aligned}
$$

where $\varsigma\left(x_{1}\right)=b x_{1}+0.5(a-b)\left(\left|x_{1}+1\right|-\left|x_{1}-1\right|\right)$. When the parameters are $\beta=10, \gamma=18, a=-4 / 3$, and $b=-3 / 4$, Chua's system has a chaotic attractor shown in Figure 7. By computation, one has $\theta=5.1623$ in view of Assumption 1. Suppose an undirected network consisting of $N=20$ nodes in terms of small word network, where the connection is given in Figure 8. Similarly, its coupling matrix is easily obtained from Figure 8 .

By Theorem 11 with coupling strength $c=85$, one has the relationship among the expectation $\alpha$, the pinning fraction $\delta$, and the pinning control gain $k$ in terms of special pinning control, which are given Figures 9 and 10, respectively. From 


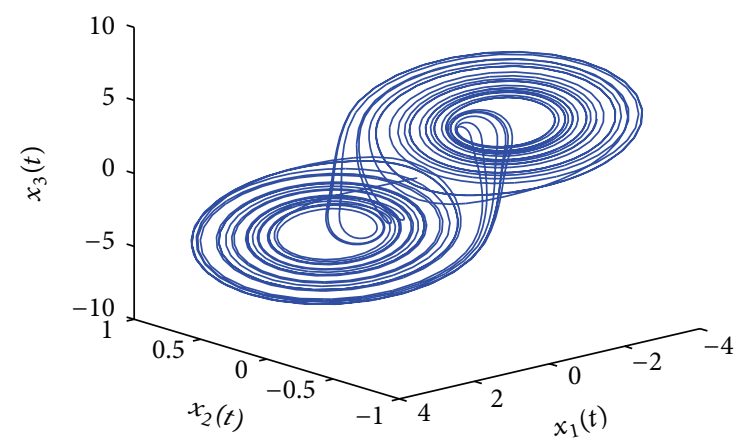

FIGURE 7: Chaotic orbits of Chua's circuit.

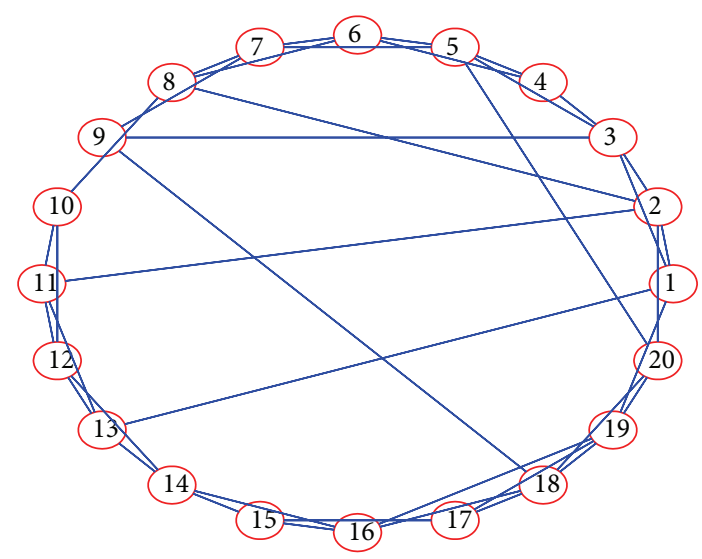

FIGURE 8: Connection of the complex network system.

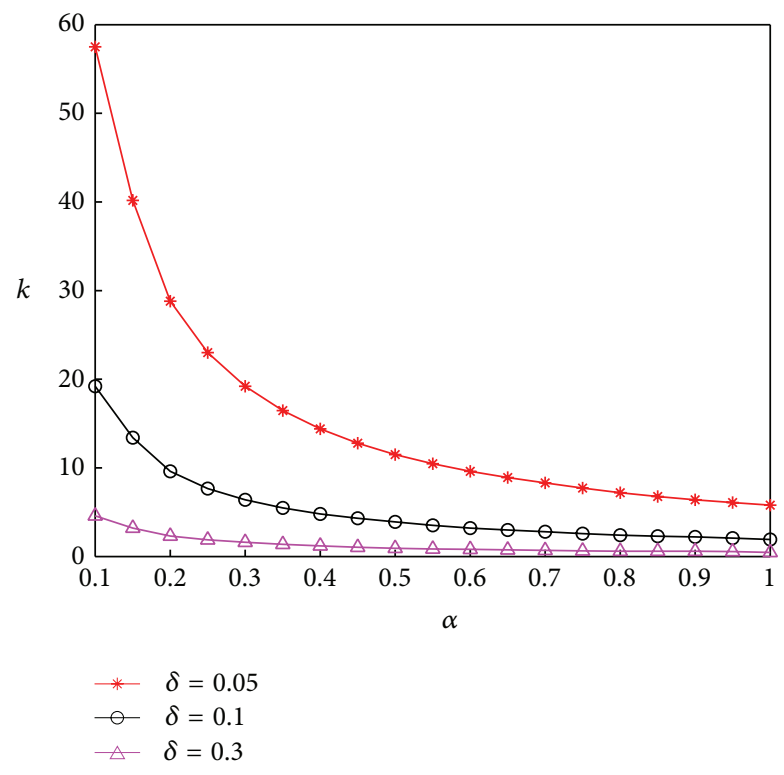

Figure 9: Correlation between $k$ and $\alpha$ with given different $\delta$.

such simulations, it is seen that larger expectation $\alpha$ results in smaller control gain $k$ with given pinning fraction $\delta$, while larger pinning fraction $\delta$ also results in smaller $k$ with given expectation $\delta$. This property is same as that in Example 15 . Let initial condition of system (55) be $x_{0}=\left[\begin{array}{lll}0.1 & -0.1 & 0.2\end{array}\right]^{T}$

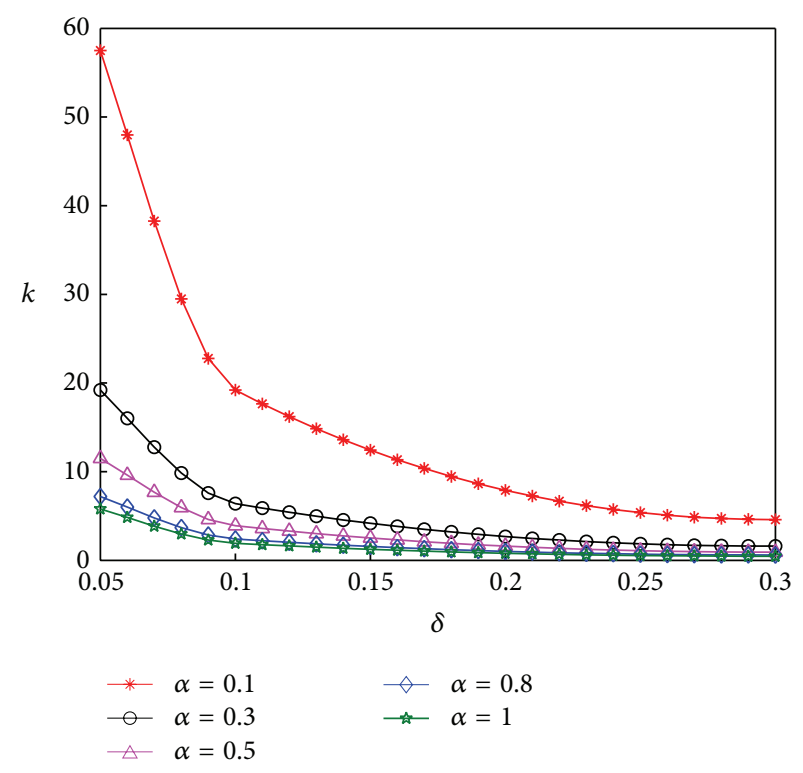

Figure 10: Correlation between $k$ and $\delta$ with given different $\alpha$.

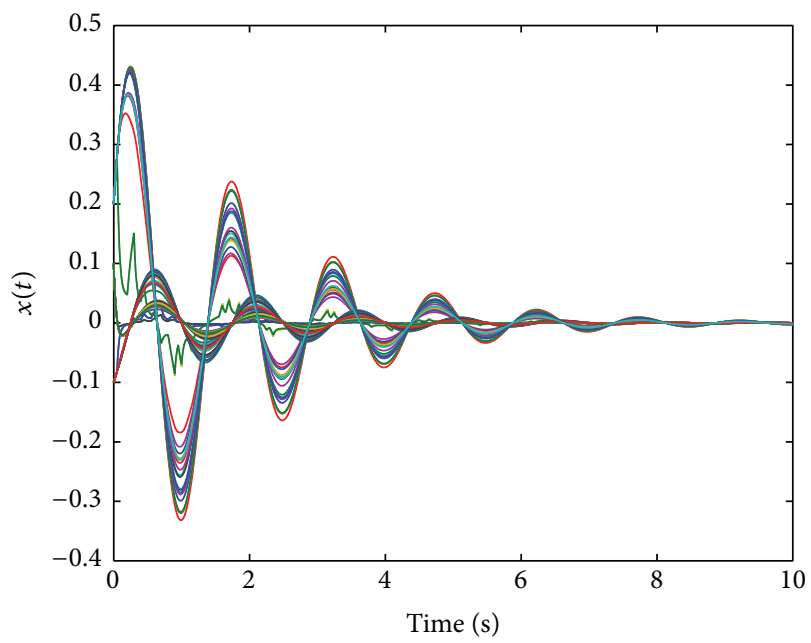

FIGURE 11: Simulation of the closed-loop system.

and $\delta=0.1, \alpha=0.7$, one has $k_{\min }=2.8$ with $k>0$ by Theorem 11. The state curve of the closed-loop system is given in Figure 11. From Figure 11, it is said that the desired pinning controller is useful. If the desired pinning controller is realized by random pinning control, we also have the corresponding simulations of correlation among $\alpha, \delta$, and $k$. Such relationships are demonstrated in Figures 12 and 13, respectively, which are quite different to the above cases. That is, the array of curves $\delta=0.05, \delta=0.1$, and $\delta=0.3$ in Figure 12 is different from those in Figures 9, 2, and 5, though there is also a consistency that larger $\alpha$ leads to smaller $k$. Accordingly, a phenomenon different from Figures 3, 6, and 10 is shown in Figure 13. For a given $\alpha$, it is seen that the value change of $k$ is not in accordance with $\delta$. Such differences come from the properties of complex network and random pinning control. 


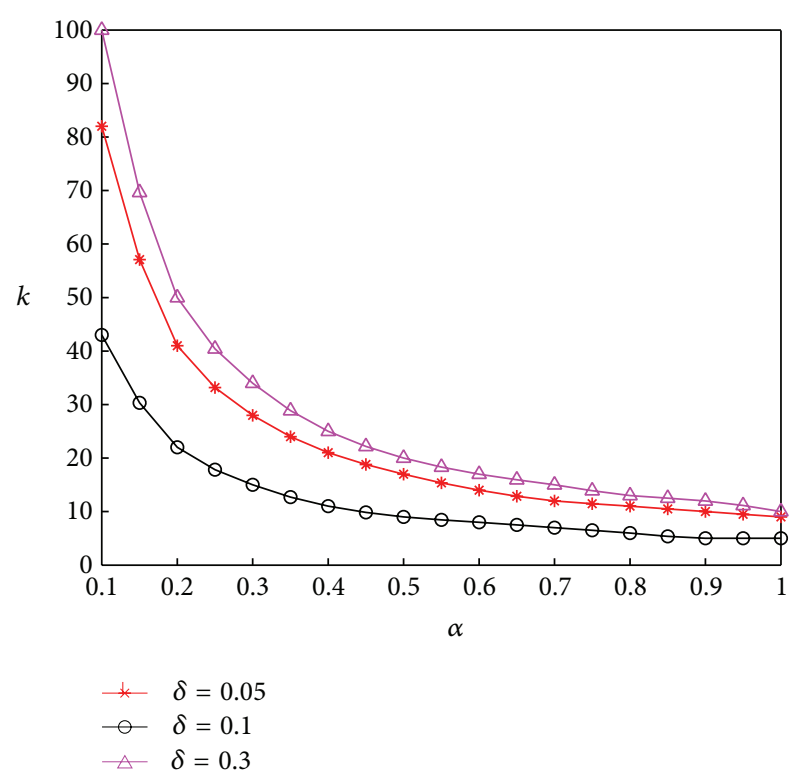

Figure 12: Correlation between $k$ and $\alpha$ with given different $\delta$.

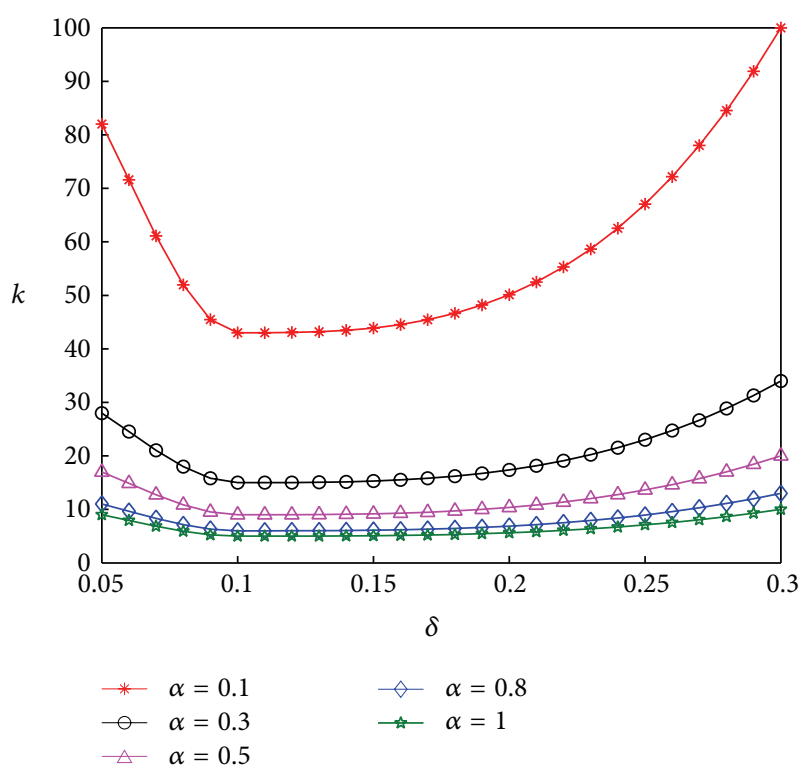

FIGURE 13: Correlation between $k$ and $\delta$ with given different $\alpha$.

\section{Conclusions}

In this paper, a new pinning method with a stochastic pinning viewpoint is proposed to investigate the control problem of multiagent systems. It has been shown that a fraction of controllers added to nodes in terms of Brownian noise perturbations can stabilize the underlying systems, whose control method is defined as "stochastic pinning control." It is also seen that the Bernoulli variable plays an essential role in realizing SPC. Based on the given method, new sufficient conditions of the expectation with uncertainty and being unknown are also established. Finally, the utility of the developed theory is illustrated by numerical examples. In this paper, there is no delay in the underlying system. When there is time delay in the controller such as [29], one may design a similar stochastic pinning controller with time delay, which will be our further topics.

\section{Conflict of Interests}

The author declares that there is no conflict of interests regarding the publication of this paper.

\section{Acknowledgments}

This work was supported by the National Natural Science Foundation of China under Grants 61104066 and 61374043, the China Postdoctoral Science Foundation funded Project under Grant 2012M521086, and the Program for Liaoning Excellent Talents in University under Grant LJQ2013040.

\section{References}

[1] R. Olfati-Saber, "Flocking for multi-agent dynamic systems: algorithms and theory," IEEE Transactions on Automatic Control, vol. 51, no. 3, pp. 401-420, 2006.

[2] M. Ji, G. Ferrari-Trecate, G. Egerstedt, and A. Buffa, "Containment control in mobile networks," IEEE Transactions on Automatic Control, vol. 53, no. 8, pp. 1972-1975, 2008.

[3] D. Dimarogonas, P. Tsiotras, and K. Kyriakopoulos, "Leaderfollower cooperative attitude control of multiple rigid bodies," Systems \& Control Letters, vol. 58, no. 6, pp. 429-435, 2009.

[4] Y. C. Cao, D. Stuart, W. Ren, and Z. Y. Meng, "Distributed containment control for multiple autonomous vehicles with double-integrator dynamics: algorithms and experiments," IEEE Transactions on Control Systems Technology, vol. 19, no. 4, pp. 929-938, 2011.

[5] Z. Meng, W. Ren, and Z. You, "Distributed finite-time attitude containment control for multiple rigid bodies," Automatica, vol. 46, no. 12, pp. 2092-2099, 2010.

[6] Y. Lou and Y. Hong, "Target containment control of multi-agent systems with random switching interconnection topologies," Automatica, vol. 48, no. 5, pp. 1165-1175, 2012.

[7] X. F. Wang and G. R. Chen, "Complex networks: small-world, scale-free and beyond," IEEE Transactions on Circuits and Systems I: Regular Papers, vol. 3, no. 1, pp. 6-20, 2003.

[8] Y. Hatano and M. Mesbahi, "Agreement over random networks," IEEE Transactions on Automatic Control, vol. 50, no. 11, pp. 1867-1872, 2005.

[9] M. Porfiri and D. J. Stilwell, "Consensus seeking over random weighted directed graphs," IEEE Transactions on Automatic Control, vol. 52, no. 9, pp. 1767-1773, 2007.

[10] W. W. Yu, G. R. Chen, Z. D. Wang, and W. Yang, "Distributed consensus filtering in sensor networks," IEEE Transactions on Systems, Man, and Cybernetics B: Cybernetics, vol. 39, no. 6, pp. $1568-1577,2009$.

[11] D. G. Xu and Z. F. Su, "Synchronization criterions and pinning control of general complex networks with time delay," Applied Mathematics and Computation, vol. 215, no. 4, pp. 1593-1608, 2009.

[12] P. Eröds and A. Rényi, "On random graphs. I.," Publicationes Mathematicae Debrecen, vol. 6, pp. 290-297, 1959. 
[13] D. J. Watts and S. H. Strogatz, "Collective dynamics of "smallworld” networks," Nature, vol. 393, no. 6684, pp. 440-442, 1998.

[14] A.-L. Barabási and R. Albert, "Emergence of scaling in random networks," Science, vol. 286, no. 5439, pp. 509-512, 1999.

[15] Y. Tang and W. K. Wong, "Distributed synchronization of coupled neural networks via randomly occurring control," IEEE Transactions on Neural Networks and Learning Systems, vol. 24, no. 3, pp. 435-447, 2013.

[16] Y. Tang, H. J. Gao, W. Zou, and J. Kurths, "Distributed synchronization in networks of agent systems with nonlinearities and random switchings," IEEE Transactions on Cybernetics, vol. 43, no. 1, pp. 358-370, 2013.

[17] X. F. Wang and G. R. Chen, "Pinning control of scale-free dynamical networks," Physica A: Statistical Mechanics and Its Applications, vol. 310, no. 3-4, pp. 521-531, 2002.

[18] X. Li, X. F. Wang, and G. R. Chen, "Pinning a complex dynamical network to its equilibrium," IEEE Transactions on Circuits and Systems I: Regular Papers, vol. 51, no. 10, pp. 2074-2087, 2004.

[19] J. H. Lü and G. R. Chen, "A time-varying complex dynamical network model and its controlled synchronization criteria," IEEE Transactions on Automatic Control, vol. 50, no. 6, pp. 841846, 2005.

[20] J. Zhou, J. A. Lu, and J. H. Lü, "Adaptive synchronization of an uncertain complex dynamical network," IEEE Transactions on Automatic Control, vol. 51, no. 4, pp. 652-656, 2006.

[21] Y. Y. Wu, W. Wei, G. Y. Li, and J. Xiang, "Pinning control of uncertain complex networks to a homogeneous orbit," IEEE Transactions on Circuits and Systems II: Express Briefs, vol. 56, no. 3, pp. 235-239, 2009.

[22] T. Liu, J. Zhao, and D. J. Hill, "Synchronization of complex delayed dynamical networks with nonlinearly coupled nodes," Chaos, Solitons \& Fractals, vol. 40, no. 3, pp. 1506-1519, 2009.

[23] P. DeLellis, M. diBernardo, and F. Garofalo, "Novel decentralized adaptive strategies for the synchronization of complex networks," Automatica, vol. 45, no. 5, pp. 1312-1318, 2009.

[24] Y. Tang, H. Gao, W. Zou, and J. Kurths, "Pinning noise-induced stochastic resonance," Physical Review E, vol. 87, no. 6, Article ID 062920, 2013.

[25] Y. Tang, Z. Wang, H. Gao, S. Swift, and J. Kurths, "A constrained evolutionary computation method for detecting controlling regions of cortical networks," IEEE/ACM Transactions on Computational Biology and Bioinformatics, vol. 9, no. 6, pp. 15691581, 2012.

[26] H. Y. Li, H. H. Liu, H. J. Gao, and P. Shi, "Reliable fuzzy control for active suspension systems with actuator delay and fault," IEEE Transactions on Fuzzy Systems, vol. 20, no. 2, pp. 342-357, 2012.

[27] H. Y. Li, J. Y. Yu, C. Hilton, and H. H. Liu, "Adaptive slidingmode control for nonlinear active suspension vehicle systems using T-S fuzzy approach," IEEE Transactions on Industrial Electronics, vol. 60, no. 8, pp. 3328-3338, 2013.

[28] T. P. Chen, X. W. Liu, and W. L. Lu, "Pinning complex networks by a single controller," IEEE Transactions on Circuits and Systems I: Regular Papers, vol. 54, no. 6, pp. 1317-1326, 2007.

[29] H. Y. Li, X. J. Jing, and H. R. Karimi, "Output-feedback-based control for vehicle suspension systems with control delay," IEEE Transactions on Industrial Electronics, vol. 61, no. 1, pp. 436-446, 2014. 


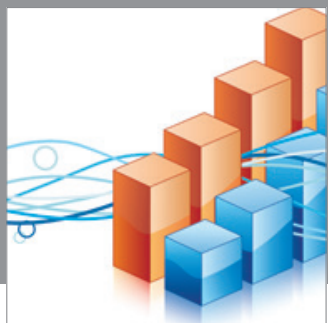

Advances in

Operations Research

mansans

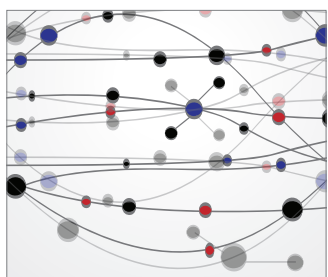

The Scientific World Journal
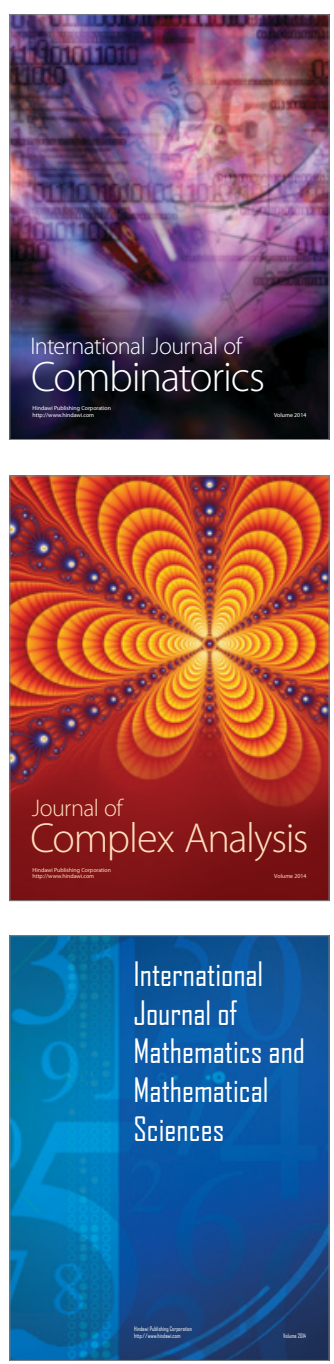
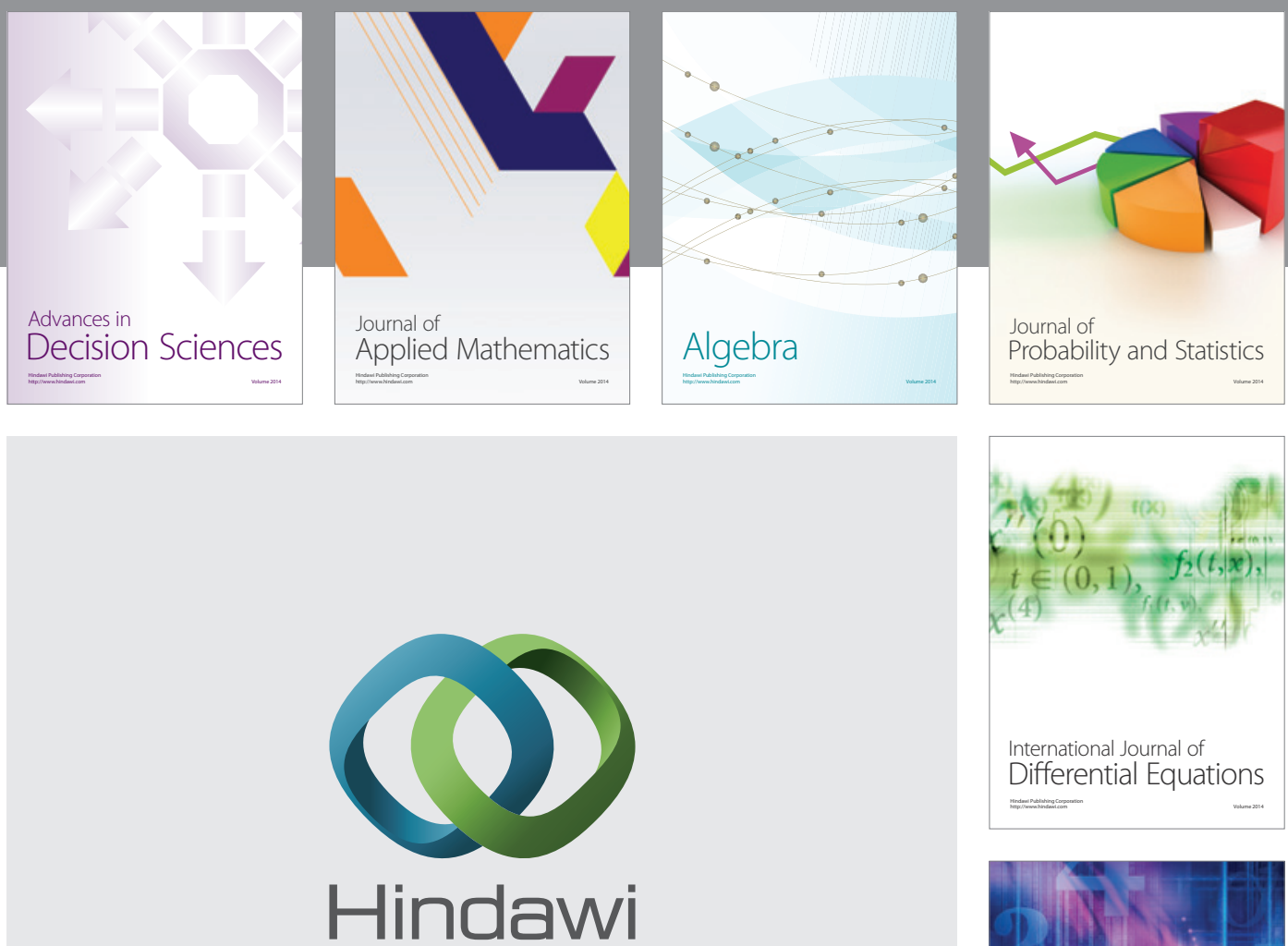

Submit your manuscripts at http://www.hindawi.com
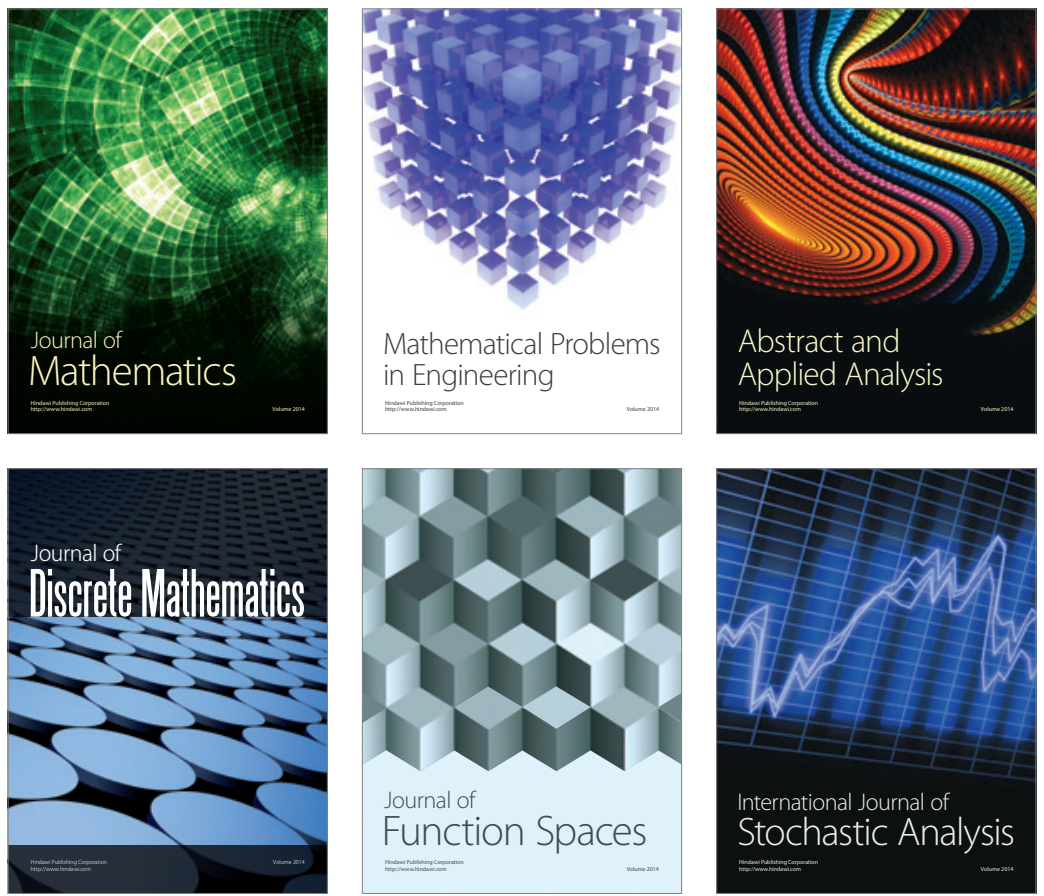

Journal of

Function Spaces

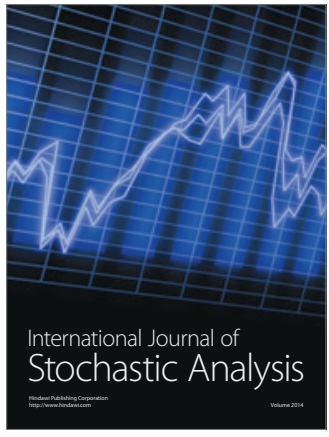

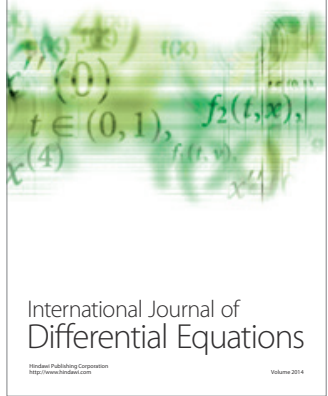
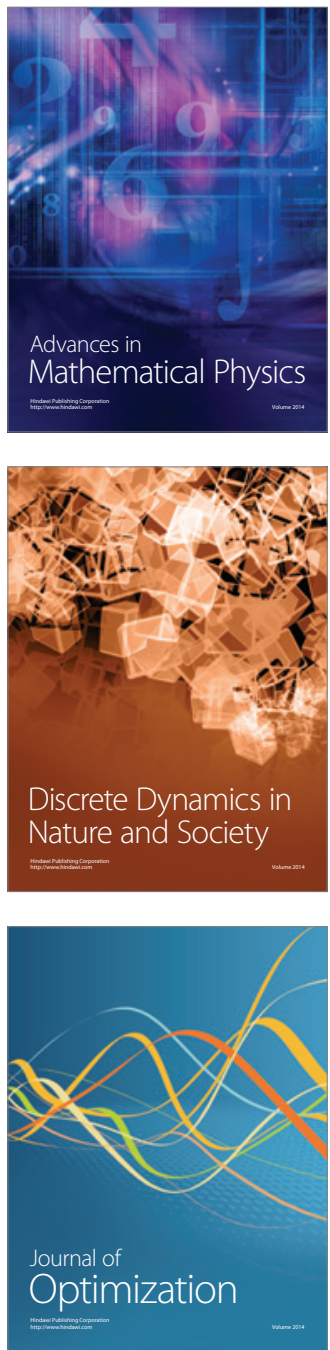